NEWS AND VIEWS

\title{
Therapeutics and prospects of Interleukin 2.
}

Nicole Lovato and Leandro Santiago Padilla.

DOI. 10.21931/RB/2019.04.04.13

Abstract: Interleukin-2 was discovered back in 1983 as an autocrine growth factor for cultured T cells and was the first biological product created through the use of recombinant DNA. IL-2 tumor immunotherapy performed the first historical clinical demonstration of the possibility to cause an effective anticancer immune reaction, mediated by cytotoxic lymphocytes activated from IL-2 stimulation. The Interleukin 2 receptor is a heterotrimeric protein that is composed of three peptide chains: the alpha chain, the beta chain and the gamma chain of the common cytokine receptor. There are 3 majors' ways of interfering with the IL-2/IL-2R to use it as treatments: Antibodies, Aptamers, and punctual mutagenesis. Recent studies have shown, that II-2 therapies for cancer, specifically targets restoring the individual's natural antitumor immune response. HIV directed treatments have demonstrated the necessity of introducing the IL-2 complemented with the patient's antiretroviral therapy.

KeyWords: Interleukin-2, cancer, HIV, immunotherapy.

\section{Introduction}

Interleukin-2 (IL-2) was discovered as an autocrine growth factor for cultured $T$ cells and was also the first cloned cytokine in 1983'. The primary function assigned to IL-2 was a strong ability to increase the proliferation and in vitro differentiation of $T$ cells $^{2}$, and it is for this reason that it was called the $T$ cell growth factor. According to its function in vitro, it was also admitted that IL-2 plays a critical role during the clonal expansion of antigen-directed $T$ cells. These findings led to clinical trials to evaluate the ability of high doses of IL-2 to activate antitumor immune responses in patients with melanoma, renal cancer and other tumors ${ }^{3}$. IL-2 was the first biological product produced through the use of recombinant DNA technology that was applied to humans with cancer or AIDS to increase the number and function of T cells. In this way it was possible to establish the bases of what has become one of the great revolutions both in the field of biomedical and clinical medicine.

Another of the functions of IL-2 is its ability to inhibit the proliferation of pro-inflammatory $T$ helper 17 cells and follicular helper $T$ cells, which are central to the production of autoantibodies ${ }^{4}$, may synergize with its effects on Treg cells in the treatment of autoimmune and inflammatory diseases. On the other hand, the challenge of using IL-2 to eliminate pathological immune responses is that the cytokine can activate the effector arm of the immune system, which carries the risk of irritating the disease. Interleukin (IL)-2 cancer therapy could be considered as the first historical immunotherapy against tumors on physio-pathological, which is developed to regulate and guide the cytokine network in an antitumor manner ${ }^{5}$ by exogenous administration of antitumor cytokines, whose endogenous production progressively decreases the cancer development ${ }^{6}$. IL-2 tumor immunotherapy performed the first historical clinical demonstration of the possibility to cause an effective anticancer immune reaction, mediated by cytotoxic lymphocytes activated from IL-2 stimulation ${ }^{5}$.

\section{Quaternary Structure Receptor}

The Interleukin 2 receptor $(\mathrm{IL}-2 \mathrm{R})$ is a heterotrimeric protein that is composed of three peptide chains: the alpha chain $(I L-2 R \alpha)$, the beta chain (IL-2R $)$, and the gamma chain of the common cytokine receptor $(\gamma \mathrm{c})$ (Figure 1$)^{1}$.
When the three chains that make up the IL-2R have been isolated, it was determined that IL-2R $\alpha$ has a low affinity to the $\mathrm{IL}-2$ receptor (binding affinity $\mathrm{kd} \sim 10 \mathrm{nM}$ ). IL-2R $\beta$ alone has a very low affinity ( $\mathrm{Kd} \sim 100 \mathrm{nM})$, and $\gamma \mathrm{c}$ alone has no detectable binding affinity for $\mathrm{IL}-2^{2}$. A complex of $\mathrm{IL}-2 \mathrm{Rb}$ and $\gamma \mathrm{c}$ bind with intermediate affinity ( $\mathrm{Kd} \sim 1 \mathrm{nM})$. A complex with three subunits IL-2R $\alpha, I L-2 R \beta$, and $\gamma c$ bind with high affinity (Kd$10 \mathrm{pM})$, which shows that the intermediate and high-affinity receptor forms are functional and cause changes in the cell when IL-2 binds to them ${ }^{3}$.

\begin{tabular}{ll} 
Subunit & Binding affinity \\
\hline IL-2 Ra & $10 \mathrm{nM}$ \\
\hline IL-2 RB & $100 \mathrm{nM}$ \\
\hline Yc & - \\
\hline IL-2Rb and $\gamma \mathbf{c}$ & $1 \mathrm{nM}$ \\
\hline IL-2Ra, IL-2 b, & $10 \mathrm{pM}$ \\
and $\boldsymbol{\gamma} \mathbf{c}$
\end{tabular}

Table 1. The binding affinity of each chain that makes up the IL-2 receptor.

\section{Signaling through the il-2 receptor}

The signaling of IL-2R contributes mainly to two of the immune responses of the CD8 $+T$ cells, which are the terminal differentiation of the effector cells in the primary responses and the memory response aspects ${ }^{2}$.

The signaling process begins when IL-2 is first captured by IL-2R $\alpha$ through hydrophobic bonds spanning a relatively weak interaction. The IL-2R $\alpha-I L-2$ complex results in a minimal conformational change in $\mathrm{IL}-2$ that drive the association with IL-2R $\beta$ and the binary complex IL-2R $\beta-I L-2$ is formed through polar interactions. It is important to emphasize that the extracellular dominium IL-2R $\alpha$ does not interact with IL$2 R \beta^{1}$. Once the IL-2R $\alpha-I L-2 R \beta-I L-2$ complex has been formed it is recruited to $\gamma c$ through a very weak interaction to IL-2 


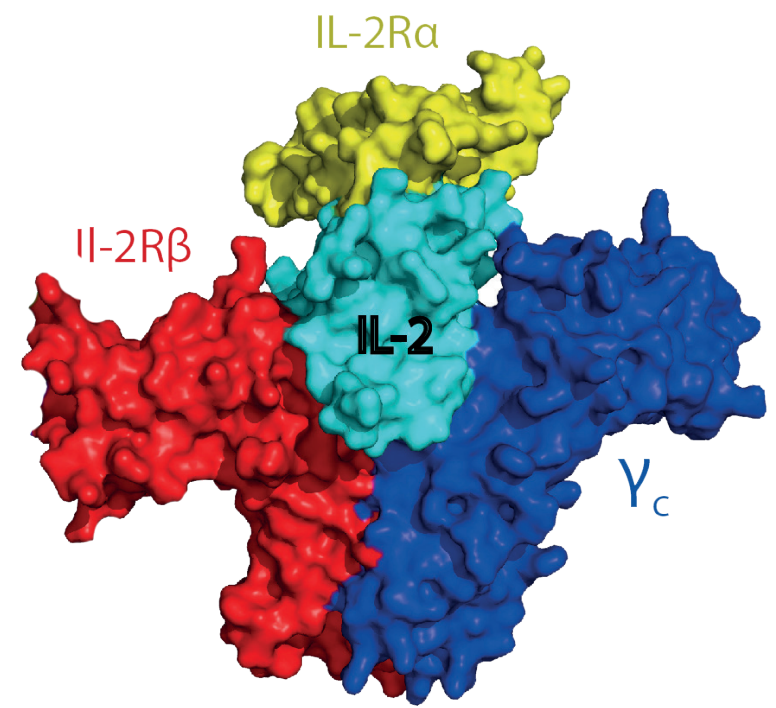

Figure 1. Surface structure of the Interleukin 2 receptor complex and its subunits. Imaged obtained using PyMOL.

and a much stronger interaction to $2 R \beta$ to produce an $\mathrm{LL}-2 \mathrm{R}$ Quaternary that is stable and of high affinity.

The creation of the high affinity IL-2-IL-2R quaternary complex leads to signal transduction, which is mediated by tyrosine kinases named Jak1 and Jak3, which in turn are associated with IL-2R $\beta$ and $\gamma c$ respectively ${ }^{4}$. There are three tyrosine residues within the cytoplasmic tail of IL-2R $\beta$ that are phosphorylated to promote the Shc adapter, then the activation of the pathways of other kinases called MAPK and PI-3K will be carried out and also the activation of a transcription factor called Stat5 that is responsible for the regulation of genes dependent on Stat5 5 . Finally, the quaternary complex of IL-2$\mathrm{IL}-2 \mathrm{R}$ is internalized briefly in the cell where IL-2, IL-2Rß, and $\gamma c$ are degraded while IL-2R $\alpha$ is recycled to the cell surface.

\section{IL2 $\mathrm{R} \alpha$ regulation}

Cellular expression of the alpha subunit is tightly regulated by three main enhancers located either upstream or downstream from the transcription unit ${ }^{6}$. Generally, resting $T$ and $B$ lymphocytes do not express the $\alpha$ subunit until they are stimulated by adjuvant cytokines. There is only a specific set on pathological conditions, like autoimmune diseases or certain hematological malignancies, that will keep the a subunit to remain expressed on the cell surface of the Lymphocytes? ${ }^{7}$. In some cases of T-cell mediated diseases the pathology is limited to only a specific area, and nowadays there are several ways to selectively target and inhibit the high-affinity IL-2 receptor in the focalized region so other IL-2R $\alpha$ expressing cells remain unharmed by the treatment ${ }^{7}$. We will discuss this article three of the primary ways of doing so.

Antibodies. Currently, a variety of monoclonal antibodies are commonly utilized to downregulate CD25 Lymphocytes clonal expansion ${ }^{8}$. Basiliximab and daclizumab are two antiIL-2R $\alpha$ mAbs that have already completed phase 3 trials for preventing acute rejection in renal transplants. Daclizumab is a humanized immunoglobulin $\mathrm{mAb}$, while basiliximab is a chimeric antibody. They both block IL-2Ra by competitive antagonism, thereby inhibiting the high-affinity receptormediated signaling while leaving unchanged IL-2 signaling by the intermediate-affinity $B$ and $Y$ receptors ${ }^{9,10}$.

Aptamers. These novel molecules are similar to antibodies when comparing their recognition capabilities in both affinity and specificity, yet these single-stranded DNA or RNA molecules elicit a much lower immunogenic response, are less toxic, and are cheaper to produce. So far, aptamer has been produced against a wide range of target molecules, including proteins or even cells, where they can act as either stimulating or inhibiting ligands ${ }^{11}$. Shahdordizadeh et al. reported, for the first time, the development of a synthetic anti-human Interleukin-2 receptor alpha (CD25) DNA aptamer (Apt51) and its functionality as a CD25 inhibitor. This study, along with the fact that aptamers have a more efficient tissue penetration, showcases and forecast the possibility of using aptamers as more efficient alternative to antibodies on IL-2 based clinical therapies ${ }^{10}$.

Mutagenesis. By introducing a particular set of mutations along the binding interface of IL-2 with the alpha subunit it is possible to disrupt their interaction. This new IL-2 mutein can, therefore, cause a decrease in CD25+ cells while still expanding CD8+ and Natural Killer cells ${ }^{12}$. A highly efficient strategy for creating this new muteins is through the recently awarded Novel Prize technique, Phage Display. Using this technique, it is possible to create enormous and diverse IL-2 mutein libraries that can, later on, be used as a selection method based on the desire ligand-receptor affinity ${ }^{13}$. There other several techniques used for the creation of new ligand mutants like protein-protein docking bioinformatics; or for creating different sets of libraries like MutS bacterial strains or error-prone PCR ${ }^{14,15}$.

\section{Cancer}

The immune system can recognize and destroy cancer cells or inhibit tumor growth by eliciting responses from both the innate and adaptive immunity. Innate immune responses are not based antigen specificity and tend to develop relatively quickly. On the other hand, responses elicited by the adaptive immune system are antigen-specific and develop more slowly. The adaptive response is mediated by both $\mathrm{T}$ (cellular response) and $\mathrm{B}$ cells (humoral response), providing the organism not only with a "fighting" mechanism but with an immune memory as well. Therefore, the rationale thinking in immune therapies against cancer is to enhance or utilize the adaptive response since it will be the one to provide a stronger and durable response ${ }^{16}$.

Cancer cells massive ability for proliferating is, in one 
way, due to the absence of an antitumor immune response. The tumor itself can produce a subset of chemical agents that will actively affect several biological pathways, inducing, at last, a lower immune response. This is why immunotherapies currently represent one of the most efficient and promising strategies against cancer when compared to chemotherapy ${ }^{17}$.

Currently, one of the main anticancer cytokines in humans is $\mathrm{IL}-2$, which represented the first historical clinical demonstration of the possibility to generate an effective anticancer immune reaction, mediated by cytotoxic lymphocytes activated from IL-2 stimulation. Using IL-2 it was able to generate LAK cells capable of destroying tumoral tissue $^{18}$.

Historically interleukin-2 has gone through numerous clinical trials, varying from high-dose treatment to low-dose treatments. High-dose treatment case studies usually had to be held on special facilities on intensive care units due to their massive side effects including capillary leak syndrome. Another side effect was cardiovascular toxicity, but the issue was fixed by using the low-dose treatment. The optimal prognosis using IL-2 obtained during trials initially, was low subcutaneous doses for 2 weeks cycles, since there wasn't any Lymphocyte count increase after this time frame, limiting the treatment application to specific metastatic tumoral growths ${ }^{19}$.

Interleukin 2 based immunotherapeutic, has faced several bumps along the way. Not only the well-known side effects of the initial trials hold it back, but the differential stimulation pathways caused by the interaction with its receptor made the scientific community to tag it as Treg Lymphocyte growth factor that may mediate the suppression of the anticancer immunity, despite its stimulatory effects on most other important immune cells ${ }^{20}$. This issue can be addressed in many ways by preventing Treg cells activation based on the previously talked mechanisms or by IL-12 and IL-21 ${ }^{17,21}$.

The new understandings regarding the antitumoral immune cytokine network are what have brought a new light into the matter. IL-2 remains as the only cytokine capable of activating a set of $\mathrm{TH} 1$ lymphocyte functions that are essential for anticancer immunity in humans, which highlights the importance of using it to restore human's inborn ability to prevent tumoral cells formation and/or growth. Since IL-2 immunotherapies aren't based on the molecule's specific ability to produce lymphocyte proliferation, but on restoring the natural antitumor immune response that was previously affected by an IL-2 deficiency or imbalance, the response to the treatment will be absolutely intrinsic to the individual understudy and prior/later monitoring is necessary for ensuring the treatment efficiency ${ }^{22,23}$.

\section{Human immunodeficiency virus (VIH)}

The Human Immunodeficiency Virus (HIV) is a retrovirus that attacks and destroys CD4 lymphocytes, which are a type of cells that are part of the immune system and are responsible for the production of antibodies to fight infections caused by these external agents ${ }^{24}$. There are antiretroviral drugs that have helped improve the quality of life of HIV positive individuals. One possible strategy is the use of interleukin-2 (IL-2) in combination with antiretroviral therapy (ART) because, without ART, IL-2 can increase the viral load up to six times its level before treatment ${ }^{25}$. These increases in viral load disappear within a month. Then, IL-2 should not be used unless it is combined with ART.

According to the Central Register of Cochrane Controlled
Trials (CENTRAL), they identified 25 eligible trials to determine the effect of IL-2 in patients with HIV. Interventions included the use of IL-2 in combination with ART compared to ART alone. Seventeen of the 21 trials reported an increase in the CD4 cell count with the use of IL-2 compared to the control with different measures. However, IL-2 has no significant effect on other clinically important positive outcomes, such as mortality rates, reduction in viral load, and opportunistic infections ${ }^{26}$.

On the other hand, in laboratory investigations, IL-2 is used to encourage the reproduction of cells infected with HIV. Then, IL-2 induces the infected cells to produce new HIV particles in large quantities. That is why some researchers were hesitant to administer this medicine to humans because the least desired is to increase the production of HIV. However, they decided to carry out the investigation. The results showed that the groups taking IL-2 combined with anti-HIV therapy (ART) and those receiving only the latter revealed similar changes in HIV levels. After a round of IL-2 treatment an explosion of HIV activity was detected, but it is not maintained, and there is no evidence that this transient rise in HIV levels is harmful ${ }^{27}$.

Although the last study was the first to reveal a favorable effect of IL-2, the differences observed between the two groups were not very large, and it is possible that the beneficial effects of IL-2 are partially eclipsed by the effect of the TAR. Moreover, both results do not support the use of IL-2 as a complement of TAR-positive because there is no improvement in the health of people infected with HIV.

\section{Conclusions}

Interleukin 2 therapeutics have proven to be efficient only under a particular set of conditions. Since the molecule's affinity for its receptor, regulates the cytokine biological effect over the lymphocytes, most of its therapeutically approach rely on interfering with the IL-2/IL-2R complex formation. When it comes to cancer, clinical studies have shown the importance of not only using a low dose subcutaneous methodology for diminishing the downsides of the treatment but also improving the previous controls for determining precise dosing. On HIV therapeutics, IL-2 therapies must be integrated with antiretrovirals therapeutics; otherwise the treatment would backfire and cause a more drastic HIV proliferation.

\section{Bibliographic references}

1. Stauber, D. J., Debler, E. W., Horton, P. A., Smith, K. A. \& Wilson, I. A. Crystal structure of the IL-2 signaling complex: Paradigm for a heterotrimeric cytokine receptor. Proc. Natl. Acad. Sci. 103, 2788-2793 (2006).

2. Malek, T. R. \& Castro, I. Interleukin-2 Receptor Signaling: At the Interface between Tolerance and Immunity. Immunity 33, 153165 (2010).

3. Kelly, G. Wire bonding techniques. Optimize hybrid speed and performance. Hybrid circuit Technol. 8, 30-32 (1991).

4. SM, R. et al. Interaction of the IL2R区 and $\bigotimes_{c}$ chain with JAK-1 and JAK-3: implications for X SCID and X CID. Science (80-. ). 266, $1042(1994)$

5. Matsumoto, B. A. et al. CIS, a Cytokine Inducible SH2 Protein, Is a Target of the JAK-STAT5 Pathway and Modulates STAT5 Activation. (2018).

6. Lin, B. B. et al. Delineation of an enhancerlike positive regulatory element in the interleukin-2 receptor alpha-chain gene. Mol. Cell. Biol. 10, 850-3 (1990)

7. Church, A. C. Clinical advances in therapies targeting the interleukin-2 receptor. QJM - Mon. J. Assoc. Physicians 96, 91-102 (2003). 
8. Zhang, Y., Jin, W. \& Cai, X. Anti-interleukin-2 receptor antibodies for the prevention of rejection in liver transplant recipients: a systematic review and meta-analysis. Ann. Med. 49, 365-376 (2017).

9. Minocha, M., Tran, J. Q., Sheridan, J. P. \& Othman, A. A. Blockade of the High-Affinity Interleukin-2 Receptors with Daclizumab High-Yield Process: Pharmacokinetic/Pharmacodynamic Analysis of Single- and Multiple-Dose Phase I Trials. Clin. Pharmacokinet. 55, 121-130 (2016)

10. Shahdordizadeh, M., Taghdisi, S. M., Sankian, M., Ramezani, M. \& Abnous, K. Design, isolation and evaluation of the binding efficiency of a DNA aptamer against interleukin 2 receptor alpha, in vitro. Int. Immunopharmacol. 53, 96-104 (2017).

11. Meyer, C. et al. Interleukin-6 receptor specific RNA aptamers for cargo delivery into target cells. RNA Biol. 9, 67-80 (2012).

12. Rojas, G., Carmenate, T. \& Leon, K. Molecular dissection of the interactions of an antitumor interleukin-2-derived mutein on a phage display-based platform. J. Mol. Recognit. 28, 261-268 (2015).

13. Vispo, N. S. et al. Displaying human interleukin-2 on the surface of bacteriophage. Immunotechnology 3, 185-193 (1997).

14. Verma, R., Wong, T. S., Schwaneberg, U. \& Roccatano, D. The mutagenesis assistant program. Methods in Molecular Biology 1179, (2014).

15. Deihim, B., Hassanzadeh, M., Shafiei-Jandaghi, N. Z., Amanlou, M. \& Douraghi, M. Characterization of the DNA mismatch repair proteins MutS and MutL in a hypermutator Acinetobacter baumannii. Microb. Pathog. 113, 74-84 (2017).

16. Carbone, D. P., Gandara, D. R., Antonia, S. J., Zielinski, C. \& PazAres, L. Non-small-cell lung cancer: Role of the immune system and potential for immunotherapy. J. Thorac. Oncol. 10, 974-984 (2015).

17. Lissoni, P. Therapy implications of the role of interleukin-2 in cancer. Expert Rev. Clin. Immunol. 13, 491-498 (2017).

18. Whittington, R. \& Faulds, D. Inter leukin-2. 46, 446-514 (1993).

19. Recchia, F. et al. Multicenter phase 2 study of interleukin-2 and 13-cis retinoic acid as maintenance therapy in advanced nonsmall-cell lung cancer. J. Immunother. 29, 87-94 (2006).

20.Chattopadhyay, S., Chakraborty, N. G. \& Mukherji, B. Regulatory T cells and tumor immunity. Cancer Immunol. Immunother. 54 1153-1161 (2005).

21. Attridge, K. et al. IL-21 inhibits T cell IL-2 production and impairs Treg homeostasis. Blood 119, 4656-4664 (2012).

22. Fumagalli, L. et al. Pretreatment serum markers and lymphocyte response to interleukin-2 therapy. Br. J. Cancer 80, 407-411 (1999).

23. Lissoni, P. et al. In vivo stimulation of IL-12 secretion by subcutaneous low-dose IL-2 in metastatic cancer patients. Br. J. Cancer 77, 1957-1960 (1998).

24.Février, M., Dorgham, K. \& Rebollo, A. CD4+T cell depletion in human immunodeficiency virus (HIV) infection: Role of apoptosis. Viruses 3, 586-612 (2011).

25. Pett, S. L., Kelleher, A. D. \& Emery, S. Role of interleukin-2 in patients with HIV infection. Drugs 70, 1115-1130 (2010).

26. Onwumeh, J., Okwundu, C. I. \& Kredo, T. Interleukin-2 as an adjunct to antiretroviral therapy for HIV-positive adults. Cochrane Database Syst Rev 5, CD009818-CD009818 (2017).

27. Enfermedad, Y. E. L. \& Vih, D. E. L. Interleuquina-2 (il-2). 2, 2-3 (2007).

Received: 2 September 2019

Accepted: 10 October 2019 